Artigo

\title{
Estado nutricional como fator prognóstico em crianças portadoras de Leucemia Linfocítica Aguda*
}

Leila N. B. Borim ${ }^{1}$ Milton A. Ruiz ${ }^{2}$

Agnes C. F. Conte 3

Beatriz Camargo ${ }^{4}$
Com o objetivo de conhecer a frequência de desnutrição proteico-calórica ao diagnóstico e avaliar a sua importância na evolução clínica de crianças portadoras de Leucemia Linfocítica Aguda, com idades inferiores a quinze anos, estudamos trinta e oito crianças com Leucemia Linfocítica Aguda e sem tratamento prévio, atendidas no Hospital de Base de São José do Rio Preto-SP, no período de Setembro/89 a Dezembro/97.

Entre as crianças, $63,2 \%$ eram do sexo masculino e $36,8 \%$ do sexo feminino $(1,7: 1,0)$, a faixa etária predominante foi de cinco a nove anos $(44,8 \%)$, seguida daquela entre um e quatro anos $(36,8 \%),{ }^{3} 10$ anos $(10,5 \%)$ e menores de um ano (7,9\%).

Ao diagnóstico, $52,7 \%$ eram eutróficos e $47,3 \%$ desnutridos (escore Z). Os índices nutricionaisavaliados foram peso/estatura, estatura/idade e peso/idade e $21 \%, 34,2 \%$ e $31,5 \%$ deles, respectivamente, estavam abaixo da normalidade. Foi observada uma alta frequência de desnutrição ao diagnóstico. Porém, na avaliação do estado nutricional, en tre os pacientes vivos e não-vivos, a presença de desnutrição não foi estatisticamente significativa.

Este estudo reforçou a evidência da necessidade de estabelecer uma rotina de avaliação nutricional ao diagnóstico, bem como, a presença de um profissional especializado em nutrição, numa unidade de oncologia pediátrica.

Rev.bras.hematol.hemoter., 2000, 22(1):47-53

Palavras-chave: desnutrição, leucemia linfocítica aguda, fatores prognósticos

\section{Introdução}

As leucemias constituem o tipo de neoplasia mais comum em crianças menores de 15 anos, correspondendo a aproximadamente um terço de todas as neoplasias humanas. Entre elas, a
Leucemia Linfocítica Aguda (LLA) é a mais comum, acometendo de $75 \%$ a $80 \%$ dos casos (1).

Os bons resultados, obtidos por diversos protocolos de tratamento da LLA em crianças, dependem de uma variedade de fatores. As características biológicas das células

1 - Departamento de Pediatria e Cirurgia Pediátrica da Faculdade Regional de Medicina de São José do Rio Preto - SP

2 - Departamento de Clínica Médica da Faculdade Regional de Medicina de São José do Rio Preto - SP

3 - Departamento de Biologia Molecular da Faculdade Regional de medicina de São José do Rio Preto - SP

4 - Departamento de Pediatria do Hospital A. C. Camargo - SP

* Hospital de Base de São José do Rio Preto - SP

Correspondência para: Leila Neves Bastos Borim

Avenida Brigadeiro Faria Lima, 5544. São José do Rio Preto. SP. CEP: 15090-000

Fone: (17) 227-2755 ramal 573. Fax: (17) 210-5051. E-mail: Inbborim@zipmail.com.br 
leucêmicas, o tratamento poliquimioterápico e as variações individuais no metabolismo das drogas, são importantes fatores que influenciam estes resultados (2). Alguns autores referem que o estado nutricional associado a várias condições, como a sócio-econômica, também podem influenciar tanto na toxicidade como na resposta ao tratamento, considerando, assim, o estado nutricional um fator prognóstico importante $(3,4)$.

A presença de desnutrição proteico-calórica ao diagnóstico nas crianças portadoras de LLA parece ser comum em determinados países, por exemplo, no México e Brasil, quando os critérios estabelecidos são os índices de peso para a idade (P/I) ou estatura para idade (E/I), $20 \%$ a $50 \%$, neste grupo de crianças, são desnutridas $(4,5$, 6). Já, em outros países, como Inglaterra, França e Estados Unidos, há diferentes resultados na avaliação do estado nutricional nestas crianças, ao diagnóstico $(7,8,9,10,11,12)$.

\section{Casuística e Métodos}

Foram incluídos neste estudo todos os portadores de LLA atendidos na Unidade de Hematologia e Oncologia Pediátrica do Hospital de Base de São José do Rio Preto, S.P., no período de setembro de 1989 a dezembro de 1997, com idade inferior a 15 anos. Os pacientes foram excluídos quando os dados de interesse do estudo, relacionados aos exames clínicos e laboratoriais dos pacientes, estavam incompletos ou quando os mesmos já haviam recebido tratamento quimioterápico ou radioterápico prévio.

Entre os pacientes diagnosticados e tratados no período de setembro de 1989 a dezembro de 1996, as informações clínicas foram obtidas de seus prontuários médicos e, daqueles que receberam diagnóstico e tratamento a partir de janeiro de 1997, os dados foram colhidos diretamente dos familiares e/ou dos pacientes. $\mathrm{Na}$ história clínica, o tempo de evolução da doença foi considerado como o período entre 0 início da queixa principal e o diagnóstico. Este tempo foi denominado precoce (até 14 dias) e não-precoce (superior ou igual a 15 dias).

Após o diagnóstico definitivo, os pacientes foram estratificados e tratados de acordo com os critérios dos protocolos de tratamentos ALLBFM 83, do Berlim-Frankfurt-München (BFM) Group, e o GBTLI-93 do Grupo Brasileiro para Tratamento de Leucemias na Infância.

Para o protocolo ALL-BFM 83, o paciente foi estratificado em um dos quatro grupos de risco: a) risco básico "baixo" (RBb); b) risco básico "alto" (RBa); c) risco médio (RM) e d) alto risco (AR). O s critérios para a estratificação mencionada incluem o tamanho do baço, o tamanho do fígado e o valor absoluto de blastos leucêmicos presentes no sangue periférico. 0 protocolo compreende quatro fases de tratamento: indução, intermediária, consolidação e manutenção.

Para o protocolo GBTLI-93, o paciente foi classificado em risco básico verdadeiro (RBV), risco básico (RB) ou alto risco (AR). Os critérios classificatórios incluem idade, leucometria inicial, tamanho do baço, tamanho do fígado, presença de massa em mediastino e acometimento do sistema nervoso central. Este protocolo, também, tem quatro fases: indução, intermediária, consolidação e manutenção.

Os índices para a classificação do estado nutricional foram baseados no peso $(\mathrm{Kg})$, estatura (cm) e idade dos pacientes, por ocasião do diagnóstico, relacionando a medida observada, com a mesma medida de uma população de referência. A população de referência utilizada foi a do National Center for Health Statistics (NCHS), que é utilizada como referência internacional e proposta pela OMS para estudos desta natureza $(13,14)$.

Três índices nutricionais foram avaliados: peso para a idade $(P / I)$, estatura para a idade $(E / I)$ e peso para a estatura (P/E). Foi aplicado 0 método do escore $Z$, o qual demonstra o quanto cada criança difere da média de referência, considerando a seguinte relação: [valor individual medido - valor da média do padrão de referência] / [desvio padrão da população de referência (NCHS) ]. A classificação utilizada para detectar a presença ou não da desnutrição energéticoprotêica e sua severidade, em todos os índices nutricionais avaliados, foi: normal $(+/-1,0 Z)$; leve $(-1,1$ a $-2,0 Z)$; moderada $(-2,1$ a $-3,0 Z)$ e severa $(<-3,0 Z)(14)$.

Para comparação dos resultados foi utilizado o teste exato de FISHER (16) e considerado 0 nível de significância de $5 \%(p<0,05)$. 


\section{Resultados}

No período de setembro de 1989 a dezembro de 1997, 43 crianças foram diagnosticadas como portadoras de LLA e receberam tratamento na unidade de Hematologia e O ncologia Pediátrica do Hospital de Base de São José do Rio Preto, S.P. Nesse período, a LLA correspondeu a $34 \%$ das neoplasias diagnosticadas. Das 43 crianças, cinco pacientes foram excluídos para compor a presente casuística por diferentes motivos que incluíram: um já havia recebido tratamento anterior em outro serviço e quatro apresentaram dados clínicos e/ou laboratoriais incompletos nos seus prontuários.

A LLA foi mais freqüente no sexo masculino $(63,2 \%)$ e a faixa etária mais freqüente foi a de $5-9$ anos (44,8\%), seguida daquela de $1-4$ anos $(36,8 \%),{ }^{3} 10$ anos (10,5\%) e $<1$ ano (7,8\%)

Entre os 38 pacientes, em 47,3\% (18 casos) foi constatado algum tipo de desnutrição, ou seja, os valores do escore $Z$ foram inferiores a 1,1 . Entre os desnutridos, quatro crianças apresentaram alterações nos índices nutricionais $P / E, E / I$ e $P / I$, cinco nos índices $E / /$ e $P / I$, duas nos índices $P / E$ e $P / I$ e três apresentaram alterações em apenas um índice (duas em $P / I$ e uma em $P / E$ ). A maior freqüência de desnutridos ocorreu quando o índice $\mathrm{E} / \mathrm{I}$ foi considerado, com $34,2 \%$ dos casos, seguido pelo índice $P / I$ com $31,5 \%$ e o índice P/E com 21\% (Tabela 1).

O tempo de evolução da doença e os dados laboratoriais obtidos na admissão ou ao diagnóstico, entre os pacientes com ou sem desnutrição, estão na Tabela 2.

Os pacientes receberam dois protocolos de tratamento, $65,8 \%$ dos casos utilizaram 0 protocolo BFM-83 e $34,2 \%$ o protocolo GBTLI93. Na avaliação entre a freqüência de pacientes vivos e não-vivos e o estado nutricional não houve diferença estatisticamente significativa entre os grupos (Tabela 3).

\section{Discussão}

A freqüência da desnutrição entre as crianças com LLA varia bastante entre os países. Alguns autores referem que ela não é freqüente, refletindo um curto espaço de tempo entre o início da doença e o seu diagnóstico $(9,10)$. Porém, nos países em desenvolvimento tem sido diagnosticada desnutrição entre $8-32 \%$ dos pacientes com neoplasias (3). Os resultados obtidos, como já mencionamos, mostraram que $47,3 \%$ dos casos deste estudo apresentavam algum tipo de desnutrição ao diagnóstico. Pode ser que os índices de desnutrição obtidos referem-se ao perfil geral dos pacientes atendidos no Hospital de Base de São José do Rio Preto.

No nosso meio, a presença de desnutrição foi encontrada em um estudo feito por Viana et al (6), em 128 crianças brasileiras portadoras de LLA. Os autores demonstraram que $21,2 \%$ dos pacientes eram desnutridos, quando $\mathrm{P} / \mathrm{I}$ foi 0 índice nutricional aplicado. Quando o índice aplicado foi $E / I, 17,4 \%$ dos pacientes apresentaram desnutrição. A alta prevalência de desnutridos não diferiu da prevalência de desnutrição entre as crianças brasileiras, quando os autores compararam os seus resultados com esta população, explicando assim 0 alto índice de desnutridos.

Yu et al (7), em um estudo com 25 crianças norte-americanas portadoras de LLA, compararam os estados nutricionais entre dois grupos de pacientes. Um grupo foi constituído por pacientes com LLA em fase inicial ou em recorrência da doença e o outro grupo por pacientes portadores de LLA em remissão. Os

Tabela 1. Freqüên cia de desnutridos segundo as classificações para $P / E, E / I$ e $P / I$

\begin{tabular}{c|c|c}
\hline \multicolumn{3}{c}{ Desnutridos } \\
\hline Escore Z & Número & $\%$ \\
$\mathrm{P} / \mathrm{E}$ & 8 & 21,0 \\
$\mathrm{E} / \mathrm{I}$ & 13 & 34,2 \\
$\mathrm{P} / \mathrm{I}$ & 12 & 31,5 \\
\hline
\end{tabular}


Tabela 2. Características clínico-laboratoriais relacionadas ao estado nutricional

\begin{tabular}{|c|c|c|c|c|c|c|}
\hline & \multicolumn{3}{|c|}{ Eutrófico } & \multicolumn{3}{|c|}{ Desnutrido } \\
\hline Características & Categoria & Número & $\%$ & Número & $\%$ & P* \\
\hline $\begin{array}{l}\text { Tempo evolução } \\
\text { precoce }\end{array}$ & $\begin{array}{l}\text { Sim } \\
\text { Não }\end{array}$ & $\begin{array}{c}5 \\
15\end{array}$ & $\begin{array}{l}25,0 \\
75,0\end{array}$ & $\begin{array}{c}3 \\
15\end{array}$ & $\begin{array}{l}16,7 \\
83,3\end{array}$ & 0,697 \\
\hline Esplenomegalia & $\begin{array}{l}\text { Sim } \\
\text { Não }\end{array}$ & $\begin{array}{c}16 \\
4\end{array}$ & $\begin{array}{l}80,0 \\
20,0\end{array}$ & $\begin{array}{c}10 \\
8\end{array}$ & $\begin{array}{l}55,5 \\
44,5\end{array}$ & 0,164 \\
\hline Hepatomegalia & $\begin{array}{l}\text { Sim } \\
\text { Não }\end{array}$ & $\begin{array}{c}15 \\
5\end{array}$ & $\begin{array}{l}75,0 \\
25,0\end{array}$ & $\begin{array}{c}14 \\
4\end{array}$ & $\begin{array}{l}77,7 \\
22,3\end{array}$ & 1,0 \\
\hline Linfoadenopatia & $\begin{array}{l}\text { Sim } \\
\text { Não }\end{array}$ & $\begin{array}{c}16 \\
4\end{array}$ & $\begin{array}{l}80,0 \\
20,0\end{array}$ & $\begin{array}{c}12 \\
6\end{array}$ & $\begin{array}{l}66,6 \\
33,4\end{array}$ & 0,468 \\
\hline $\begin{array}{l}\text { Morfologia- } \\
\text { Sistema FAB }\end{array}$ & $\begin{array}{l}L_{1} \\
L_{2}\end{array}$ & $\begin{array}{l}17 \\
3\end{array}$ & $\begin{array}{l}85,0 \\
15,0\end{array}$ & $\begin{array}{c}12 \\
6\end{array}$ & $\begin{array}{l}66,6 \\
33,4\end{array}$ & 0,260 \\
\hline $\begin{array}{l}\text { Comprometimento } \\
\text { SNC }\end{array}$ & $\begin{array}{l}\text { Sim } \\
\text { Não }\end{array}$ & $\begin{array}{c}7 \\
13\end{array}$ & $\begin{array}{l}35,0 \\
65,0\end{array}$ & $\begin{array}{c}6 \\
12\end{array}$ & $\begin{array}{l}33,4 \\
66,6\end{array}$ & 1,0 \\
\hline $\begin{array}{l}\text { Hemoglobina } \\
f 8 \mathrm{~g} / \mathrm{dl}\end{array}$ & $\begin{array}{l}\text { Sim } \\
\text { Não }\end{array}$ & $\begin{array}{c}12 \\
8\end{array}$ & $\begin{array}{l}60,0 \\
40,0\end{array}$ & $\begin{array}{c}11 \\
7\end{array}$ & $\begin{array}{l}61,1 \\
38,9\end{array}$ & 1,0 \\
\hline $\begin{array}{l}\text { Leucócitos } \\
350 \times 10^{9} \mathrm{cel} / \mathrm{L}\end{array}$ & $\begin{array}{l}\text { Sim } \\
\text { Não }\end{array}$ & $\begin{array}{c}4 \\
16\end{array}$ & $\begin{array}{l}20,0 \\
80,0\end{array}$ & $\begin{array}{c}4 \\
14\end{array}$ & $\begin{array}{l}22,3 \\
77,7\end{array}$ & 1,0 \\
\hline $\begin{array}{l}\text { Plaquetas } \\
<150 \times 10^{9} \mathrm{cel} / \mathrm{L}\end{array}$ & $\begin{array}{l}\text { Sim } \\
\text { Não }\end{array}$ & $\begin{array}{c}18 \\
2\end{array}$ & $\begin{array}{l}90,0 \\
10,0\end{array}$ & $\begin{array}{c}13 \\
5\end{array}$ & $\begin{array}{l}72,2 \\
27,8\end{array}$ & 0,222 \\
\hline Blastos periférico & $\underset{N a ̃ o}{S i m}$ & $\begin{array}{c}17 \\
3\end{array}$ & $\begin{array}{l}85,0 \\
15,0\end{array}$ & $\begin{array}{l}13 \\
5\end{array}$ & $\begin{array}{l}72,2 \\
27,8\end{array}$ & 0,438 \\
\hline Total & & 20 & 100,0 & 18 & 100,0 & \\
\hline
\end{tabular}

*Teste de Fisher

Tabela 3. Frequência de pacientes vivos e não-vivos em relação aos índices nutricionais $P / E, E / /$ e $P / I$

\begin{tabular}{l|c|c|c|c|c|c}
\hline & \multicolumn{4}{|c|}{ Vivos } & \multicolumn{3}{c}{ Não-vivos } \\
\hline Variável & Categoria & Número & $\%$ & Número & $\%$ & P* \\
nutricional & Eutrófico & 24 & 82,7 & 6 & 66,6 & 0,363 \\
& Desnutrido P/E & 5 & 17,3 & 3 & 33,4 & \\
& Eutrófico & 19 & 65,5 & 6 & 66,6 & 1,0 \\
& Desnutrido E/I & 10 & 34,5 & 3 & 33,4 & \\
& Eutrófico & 22 & 75,9 & 4 & 44,4 & 0,108 \\
& Desnutrido P/I & 7 & 24,1 & 5 & 55,6 & \\
\hline Total & & 29 & 100,0 & 9 & 100,0 & \\
\hline
\end{tabular}

*Teste de Fisher 
autores observaram índices bioquímicos indicativos de desnutrição apenas no grupo de pacientes portadores de LLA em fase inicial ou em recorrência, sugerindo que os portadores de leucemia, com doença ativa, apresentam algum grau de desnutrição. Reilly et al (12) encontraram uma prevalência de desnutridos, estatisticamente significativa, entre crianças inglesas portadoras de LLA, e ressaltaram a importância de uma rotina de avaliação nutricional entre os pacientes.

Já, Uderzo et al (9), em um estudo desenvolvido no San Gerardo Hospital-Monza (Itália), compararam o estado nutricional ao diagnóstico de 173 crianças portadoras de LLA com o de 307 crianças com uma doença benigna aguda e concluíram que, as primeiras, não apresentaram uma depleção nutricional importante. Delbcque-Boussard et al (11), em um estudo com 15 crianças portadoras de LLA, que receberam tratamentos no University Hospital of Lille (França), também não encontraram depleções nos estados nutricionais.

As características clínicas não diferiram entre os pacientes eutróficos e desnutridos e foram semelhantes à literatura $(17,18)$. Q uanto as manifestações laboratoriais, as frequências na concentração de hemoglobina menor ou igual a $8,0 \mathrm{~g} / \mathrm{dl}$ e no comprometimento do sistema nervoso central ao diagnóstico foram elevadas, quando comparadas com dados de outros estudos $(17,18,19)$, porém, elas não diferiram entre os dois grupos.

Algumas casuísticas, já descritas, foram analisadas com a finalidade de estudar os possíveis efeitos adversos da desnutrição na resposta quimioterápica e no prognóstico do paciente com LLA. Na avaliação do estado nutricional entre os pacientes vivos e não-vivos não houve diferença estatisticamente significativa, contudo, em relação ao índice nutricional $\mathrm{P} / \mathrm{I}$, houve uma maior freqüência de óbitos entre os desnutridos. Weir et al (21) sugerem que apenas a desnutrição proteico-calórica grave, freqüente nos países em desenvolvimento, deve ser considerada como um fator prognóstico entre as crianças pordadoras de LLA, pois, observaram que a desnutrição proteico-calórica leve não foi um fator prognóstico importante, quando avaliaram 1025 crianças inglesas, portadoras de LLA baixo risco, com idades entre zero e quinze anos.

A desnutrição associada a outros fatores sócio-econômicos, tais como: renda mensal, acesso à comunicação, ao transporte e à educação, parece afetar a resposta terapêutica das crianças portadoras de LLA. Viana et al (4), sugerem que as condições sócio-econômicas são mais importantes que o estado nutricional. Em um estudo com 167 crianças brasileiras portadoras de LLA, os autores avaliaram a presença de desnutrição e o índice sócioeconômico (SES = renda mensal + gasto energético) e observaram uma sobrevida livre de doença em 8 anos de $30 \%$ entre as crianças eutróficas e com SES $<3,1$ e de $58 \%$ entre as crianças eutróficas e com SES $>3,1(p<0,0002)$, porém, entre as eutróficas e as desnutridas e com SES <3,1, a sobrevida livre de doença em 8 anos não apresentou uma diferença estatisticamente significativa $(p=0,1)$.

Estudos, principalmente em países em desenvolvimento, têm mostrado que a desnutrição é um fator prognóstico importante. LobatoMendizábal et al (5) demonstraram que o prognóstico das crianças com LLA está relacionado com fatores sócio-econômicos e que a desnutrição é o principal fator responsável pelo prognóstico desfavorável, no grupo de baixo índice sócio-econômico. Daly et al (20) estudaram a resposta ao tratamento quimioterápico, cirúrgico e/ou radioterápico entre 140 pacientes norte-americanos desnutridos e portadores de neoplasias. Os autores demonstraram que a imunidade celular apresenta uma correlação direta e significativa com os parâmetros utilizados na avaliação dos índices nutricionais e que os pacientes com uma resposta imunológica deficiente apresentam uma freqüência maior de desnutrição grave, níveis séricos de albumina baixos, linfopenia mais acentuada, resposta inferior ao tratamento quimioterápico e morbidade maior no período pós-cirúrgico. Reilly et al (8) estudaram as respostas terapêuticas e as evoluções clínicas de 70 crianças inglesas portadoras de LLA, com o objetivo de avaliar a relação do índice $P / E$ ao diagnóstico e o seu valor prognóstico. Os autores relataram que os episódios de recorrências foram mais freqüentes entre os pacientes que apresentavam os índices $\mathrm{P} / \mathrm{E}$ inferiores à - 0,5 ao diagnóstico. Viana et al 
(6) avaliaram o tempo de remissão em relação a presença ou não de desnutrição entre 128 crianças brasileiras portadoras de LLA e encontraram uma sobrevida livre de doença em cinco anos de $83 \%$ entre os pacientes eutróficos e de $26 \%$ entre os pacientes com 0 índice $E / I$ inferior ao normal $(p<0,001)$.

Sabendo-se que a desnutrição proteicocalórica é freqüente entre as crianças portadoras de LLA, principalmente nos países em desenvolvimento $(5,6,8)$ e, que ela associada a fatores sócio-econômicos e culturais, pode influir negativamente no tratamento e no prognóstico dos pacientes ( 3 , 4), é necessário estabelecer uma rotina para a avaliação nutricional ao diagnóstico e durante o tratamento de todos os pacientes de uma unidade de oncologia pediátrica, bem como, o acompanhamento nutricional realizado por profissional especializado, para prevenção ou intervenção terapêutica.

\section{Nutritional status as prognostic factors in children with acutelymphoblastic leukemia} Leila N. B. Borim, Milton A. Ruiz, Agnes C. F. Conte, Beatriz Camargo

\section{Abstract}

This study evaluated 38 children with Acute Lymphoblastic Leukemia, under 15 years old, without previous therapy, seen at the Hospital de Base, Medical School of Saõ J osé do Rio Preto, S.P., during the period between September, 1989 to December, 1997. Nutritional status were compared as well as malnutrition at diagnosis with patients survival were compared.

The results showed the following incidences: males $63.2 \%$, females $36.8 \%$. The stratification by age showed greater incidencein the age group of $5-9$ years $(44.8 \%)$, followed by $1-4$ years $(36.8 \%)$, and older than 10 years $(10.5 \%)$ and the last group, younger than 1 year (7.9\%).

The study of nutritional status showed that $52.7 \%$ of children were wellnourished and $47.3 \%$ were undernourished at diagnosis (score $Z$ ). The anthropometric measurements showed that 21\% of children were malnourished according to the weight for height index, $34.2 \%$ according to the height for age index, and $31.5 \%$ according to the weight for age index at diagnosis.

Malnutrition was commonly present at diagnosis. However, the survival was not influenced by malnutrition.

The present stu dy an alysi s has demonstrated the necessity to improve the nutritional assessment at diagnosis as well as nutritional intervention. Rev.bras.hematol.hemoter., 2000, 22(1):47-53

Key words: malnutrition, acute lymphoblastic leukemia, prognostic factors

\section{Referências bibliográficas}

1. Parkin D.M., Stiller C.A., Draper G.J. et al. The international incidence of childhood cancer. IntJ Cancer 1988a; 42: 511-520.

2. Pui C-H., Evans W.E. Acute lymphoblastic leukemia. N Eng J Med. 1998; 339: 605-615.

3. Gómez-Almaguer D., Ruiz-Argüelles G.J., Ponce-de-León S. Nutritional status and socio-economic conditions as prognostic factors in the outcome of therapy in childhood acute lymphoblastic leukemia. Int J. Cancer 1998; Supl 11: 52-55.

4. Viana M.B., Fernandes R.A.F., De Carvalho R.I. et al. Low socioeconomic statusisa strong independent predictor of relapse in childhood acu telymphoblastic leukemia. Int. J. Cancer 1998; Supl 11: 56-61.

5. Lobato-Mendizábal E., Ruiz-Argüelles G.L., Ganci-Cerrud G. Efecto del estado socioeconômico sobrela respuesta terapéutica de niños com leucemia aguda de riesgo habitual. Neoplasia 1991; 8: 161-165.

6. Viana M.B., Murao M., Ramos G. et al. Malnutrition as a prognostic factor in lymphoblastic leukaemia: a multivariate analysis. Arch Dis Child 1994; 71; 304-310.

7. Yu L.C., Kuvibidila S., Ducos R. et al. Nutritional status of children with leukemia. Med Pediatr Oncol 1994; 22: 73-77.

8. Reilly J.J., Odame I., McColl J.H. et al. Does weight for height have prognostic significance in children with acutelymphoblastic leukemia. Am J Ped Hematol Oncol 1994; 16: 225-230.

9. Uderzo C., Rovelli A., Bonomi M. etal. Nutritional statusin untreated children with acuteleukemia ascompared with children withoutmalignancy. J Pediatr Gastroenterol Nutr 1996; 23: 34-37. 
10. Brennan B.M., Thomas A.G. Nutritional statusin children with acute leukemia [letter]. J Pediatr Gastroenterol Nutr 1997; 25: 248.

11. Delbecque-Boussard L., Gottrand F., Ategbo $S$. et al. Nutritional status of children with acutelymphoblastic leukemia: a longitudinal study. Am J Clin Nutr 1997; 65: 95-100.

12. Reilly J.J., Weir J., Gibson B.E. Prevalence of protein-energy malnutrition at diagnosis in children with acute lymphoblastic leukemia. J Pediatr Gastroenterol Nutr 1999; 29: 194-197.

13. Gorstein J., Sillivan K., Yip R. et al. Issues in the assessment of nutritional status using anthropometry. Bull World Health Organ 1994; 72: 273-283.

14. Silva M.K.S., Félix D.S. Uso da antropometria na avaliação do estado nutricional. Rev Bras Clin 1998; 13: 74-80.

15. Mora J.0. A new method for estimating a standardized prevalence of child malnutrition from anthropometric indicators. Bull World Health Organ 1989; 67: $133-142$.

16. Runyon R.P, editor. Fundamentes of statistics in the biological, medical and health sciences. $1^{\circ}$ ed. Boston: PWS Publishers; 1985.
17. Azevedo A., Loureiro P., Freire A.D. et al. Acute lymphoblastic leukaemia: experience of treatment in northeast of Brazil (19851990) [abstract]. Med Pediatr Oncol 1992; 20: 406.

18. Margolin J.F., Poplack D.G. Acute Lymphoblastic Leukemia. In: Pizzo PA, Poplack DG, editores. Principles and Practice of Pediatric Oncology. $3^{\circ} \mathbf{e d .}$ Philadel phia: JB Lippincontt Company; 1997. p. 409-462.

19. Kreuger A., Gustafsson G., Hertz H. et al. CNS-involvement in childhood ALL [abstract]. Med Pediatr Oncol 1992; 20: 385.

20. Daly J.M., Dudrick S.J., Copeland E.M. Evaluation of nutritional indices as prognostic indicators in the cancer patient. Cancer 1979; 43: 925-931.

21. Weir J., Reilly J.J., McColl J.H. et al. No evidence for an effect of nutritional status at diagnosison prognosisin children with acute lymphoblastic leukemia. J Pediatr Hematol Oncol 1998; 20: 534-538.

Recebido: 29/02/2000

Aceito: $10 / 04 / 2000$ 